\title{
Turbomachinery Design Quality Checks To Avoid Friction Induced Structural Failure
}

\author{
Abstract by \\ Jerry H. Moore, Design Project Engineer \\ Liquid Space Propulsion \\ Pratt \& Whitney \\ West Palm Beach, Florida 33410
}

A unique configuration of the P\&W SSME Alternate Fuel Turbopump turbine disk/blade assembly (Figure 1), combined with a severe thermal environment (Figure 2), resulted in several structural anomalies that were driven by frictional contact forces. Understanding the mechanics of these problems provides new quality checks for future turbo machinery designs.

During development testing in 1997 of the SSME alternate fuel turbopump at Stennis Space Center, several potentially serious problems surfaced with the turbine disk/blade assembly that had not been experienced in extensive earlier testing. Changes to the operational thermal environment were noted based on analytical prediction of modifications that affected performance and on stationary thermal measurements adjacent to the rotor assembly. A detailed structural investigation was required to reveal the mechanism of distress induced by the change. The turbine disk experienced cracking in several locations due to increased thermal gradient induced stress during start and shutdown transients. This was easily predictable using standard analysis procedures and expected once the thermal environment was characterized. What was not expected was the curling of a piston ring used for blade axial retention in the disk., indentation of the axial face of the blade attachment by a spacer separating the first and second stage blades, and most significantly, galling and cracking of the blade root attachment that could have resulted in blade release (Figure 1).

Past experience, in gas turbine environments, set a precedent of never relying on friction for help and to evaluate it only in specific instances where it was obvious that it would degrade capability. In each of the three cases above, friction proved to be a determining factor that pushed the components into an unsatisfactory mode of operation. The higher than expected temperatures and rapid thermal transients combined with friction to move beyond past experience. The turbine disk/blade assembly configuration contributed to the potential for these problems to occur by limiting the radial deflection from thermals and centrifugal loading. The cooled solid bore configuration was chosen to improve rotordynamic stability by limiting the length of rotor overhang while still protecting the roller bearing by maintaining zero slope under the inner race (Figure 1). During a start transient, the rim area of the disk heats rapidly and expands axially and circumferentially and requires corresponding radial and axial growth of the disk to maintain relative positioning of the disk, blades, spacers and retainer rings. The stiffness, large thermal mass, and bore cooling flow combine to severely limit the disk rim radial growth which results in the potential for relative movement between these parts. Friction then becomes a player in the determination of component stress. 
The forward and rear piston rings were designed with an angle cut so the ring could grow circumferentially more than the restraining disk structure by sliding and overlaping itself. The rear ring experienced yielding and permanent curling deformation while the forward ring retained its original shape. Non-linear (plastic) analysis predicted a yielding condition only when the ring was restrained from circumferential sliding (Figure 3 ). The significant parameter controlling sliding was the length of circumferential support with the front ring being supported for $66 \%$ of the circumference versus $25 \%$ for the rear ring. Higher contact loading combined with more ring deflection between supports that created a ramp effect, resulted in a locked condition for the rear ring. The front ring slipped and remained un-deformed. Reduced temperatures lowered the potential for curling in the ring and a retaining pin was applied to restrain the ring from curling and becoming disengaged with its retaining slot (Figure 4 ).

The second stage blade experienced an indentated surface from axial contact with the interstage spacer (Figure 5). The spacer maintains relative axial position of the blades in the disk slot and is made from the same material as the disk for thermal compatibility. However, the rapid flowpath temperature increases during start combined with high " $\mathrm{H}$ " from the high pressure Hydrogen environment and large areas of surface exposure, resulted in extremely fast heating of the spacer. The adjacent disk lug, with less direct flowpath exposure and integral contact with the large mass of the main body of the disk (conduction route), responds much slower. The spacer thus experiences a larger axial growth than the disk lug which results in pressure on the adjacent blades that attempts to move them axially in the slot. The blades have very high attachment contact loading, relative to the spacers, due to centrifugal loading and therefore are subject to large frictional forces that restrain their axial movement. Analysis of this transient response, with the blades axially restrained, resulted in loading predicted to yield the blades at the blade/spacer interface (Figure 6). A significant reduction of the friction from blade contact loading was not an option, so

lowering of flowpath temperature was seen as the main corrective action to reduce the transient thermal mismatch of the parts.

The blade attachments experienced cracking induced by heavy galling at the edge of the contact zone. Transient analysis, with the higher temperature environment being experienced at the time of the cracking, found that the rapid thermal growth of the attachments, relative to the disk body, resulted in significantly higher contact loading between the disk and blade. This was due to a phenomena where the circumferential growth of the attachments relative to the live disk resulted in a tendency for the blade to slide inward (negative slip) (Figures $7 \& 8$ ) on the contact surfaces relative to the disk. In normal rotor operation, disk thermal and centrifugal loading result in radial disk deflection that provides disk attachment circumferential opening and results in blade movement outward (positive slip) along the contact surfaces. Friction in the normal positive slip mode reduces normal contact loading while friction in the negative slip mode increases normal contact loading (Figure 9). The increased normal loading from negative slip and resultant increased sliding friction Figures $10 \& 11$ ), produced a galled surface that generated small cracks that subsequently began to propagate. The solution to 
this problem was to reduce temperatures and to reduce contact friction by applying a thin layer of gold on the contact surfaces.

The measured and predicted environment matched results of structural analysis that confirmed that this environment was required to produce the observed damage. Together they provided convincing evidence that the root cause was reached in the investigation and proper corrective action taken. The primary lesson learned from this study is that the potential exists even in gas turbines for situations where negative slip might occur in attachments during fast transients. When this is combined with friction, increased contact loading can occur that can result in severe material surface damage and eventual component failure. This condition can be predicted easily using a simple 2D model of the attachment geometry with boundary conditions from a $2 \mathrm{D}$ body-of-revolution model of the disk under transient thermal conditions. The precedent of never relying on friction for help remains a good approach, but this study emphasizes that more attention needs to be directed to situations where friction may induce un-welcomed effects. The rocket engine rapid transient thermal environment, combined with a unique rotor configuration, resulted in mechanical anomalies unexpected based on previous gas turbine experience base. While these effects may not have been evident in past turbomachinery designs, the requirement for higher performance, higher temperatures, and lower weight may drive rotor designs to configurations and environmental conditions that may respond in a similar matter to the parts in this study. Therefore, the lessons learned should be applied to gas turbine as well as rocket turbomachinery development.

JHM 1]-19-98 TECHPAPR.DOC

\section{SSME-AT HPFTP TURBINE DISK ASSEMBLY}

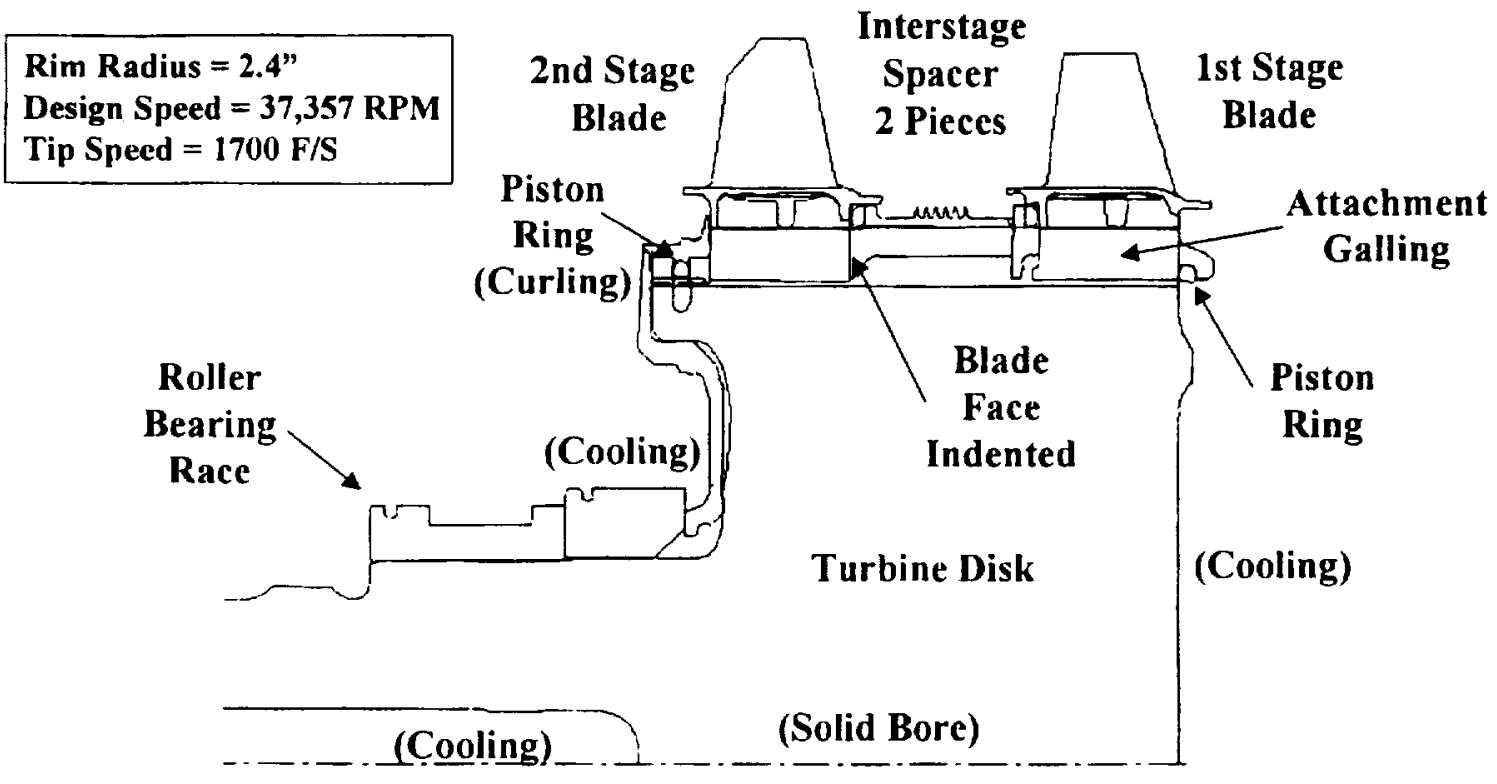

Figure 1

JIM II-16-98 DISKSKET.PPT 
SSME-AT FUEL TURBOPUMP

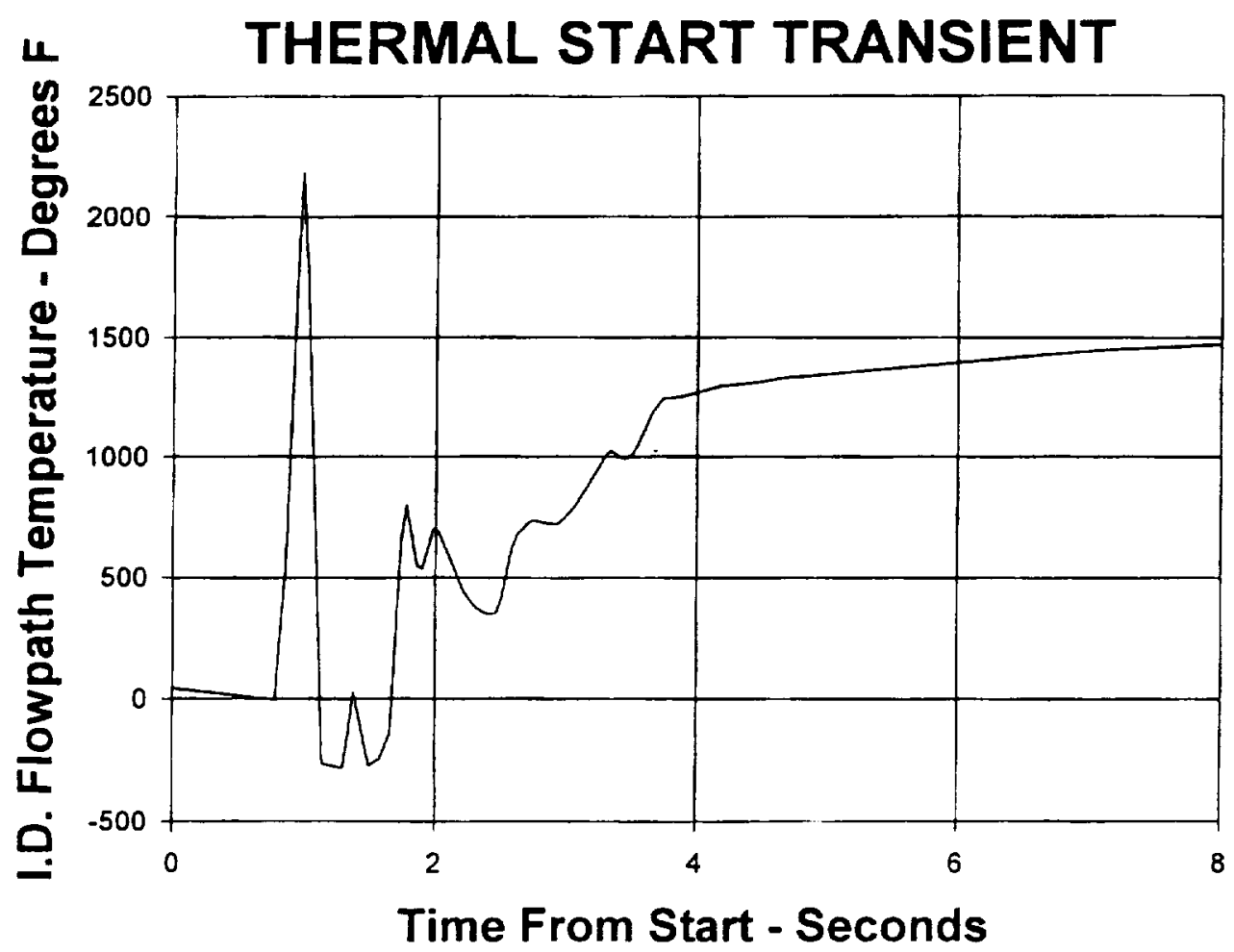

Figure 2

JHM 11-16-98 STARTTR.JNB

TURBINE BLADE AXIAL RETENTION RING Analytical Prediction Of Ring Permanent Deformation

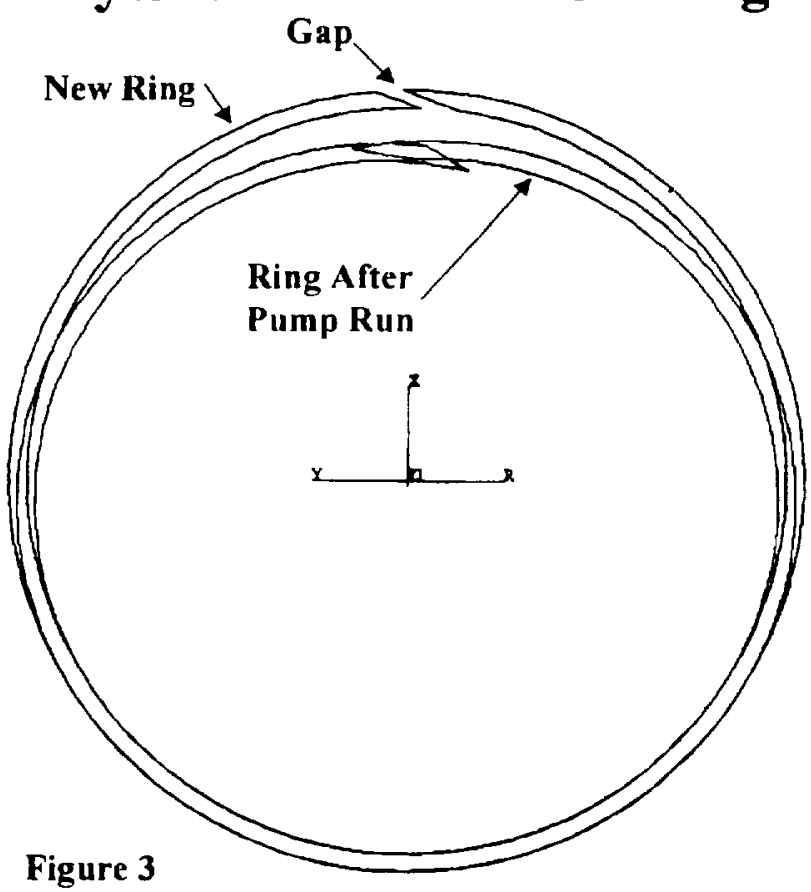

Prediction

-Initial Slot Gap $=0.120$

$\cdot$ Residual Slot Gap $=\mathbf{- 0 . 5 8 0}$

Measured

$\cdot$ Slot Gap $=\mathbf{- 0 . 7 8 0}(\mathrm{Max})$

JHM 11-18-98 PAPFIG3.PPT 


\section{TURBINE BLADE AXIAL RETENTION RING}

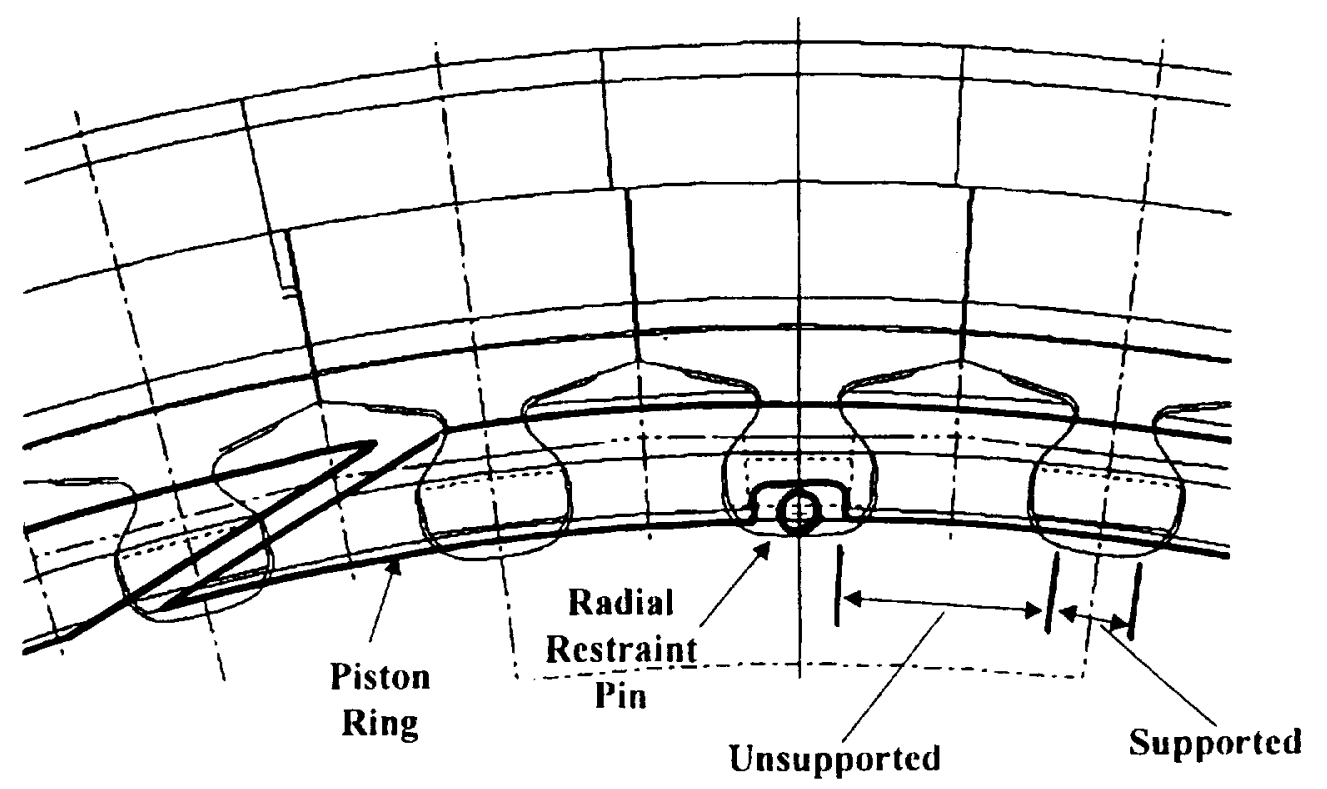

Figure 4

JHM 11-18-98 PAPFIG4.PPT

\section{2nd TURBINE BLADE ATTACHMENT}

\section{Depression From Axial Contact With Interstage Spacer}

Figure 5

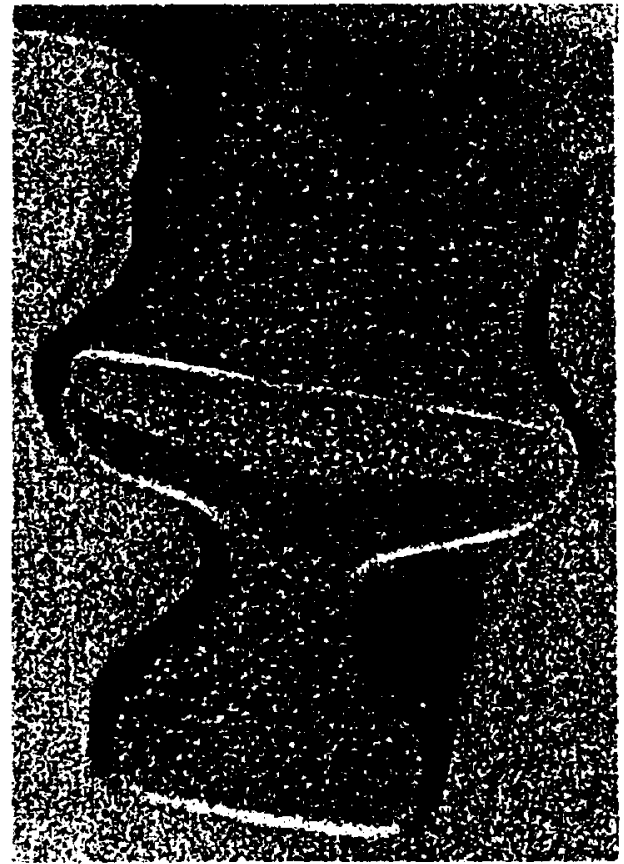




\section{HPFTP-AT TURBINE}

\section{Blade Axial Contact Face Depression Analysis Predicts Blade Depression Scenario}

- Radial contact loading in attachment is consistent with axial lockup

- Axial load to overcome friction (0.3) is $>6,000 \#$

- Maximum axial loading predicted between $4 \& 8$ seconds after start

- Higher than design intent temperatures produce excessive axial loading

- Delta axial thermal growth between spacer and disk

- Blades predicted to yield first - spacer should not show depression

- Spacer material has higher yield capability

- 2nd blade predicted to yield before 1 st

- Smaller contact area - higher stress level - O.D. loading pattern

- Supported by measured depressions

- Increase in contact area at $\mathbf{2 n d}$ blade is not effective in reducing contact stress

- Radial thermal gradient drives O.D. contact pattern

\begin{tabular}{|c|c|c|c|c|c|c|}
\hline $\begin{array}{c}\text { Analysis } \\
\text { Conditions }\end{array}$ & $\begin{array}{c}\text { Contact } \\
\text { Load }\end{array}$ & Temperature & $\begin{array}{c}\text { 1st Blade/Spacer } \\
\text { Stress }\end{array}$ & $\begin{array}{c}\text { 2nd Blade/Spacer } \\
\text { Stress }\end{array}$ & $\begin{array}{c}\text { Blade Compressive } \\
\text { Yield Strength }\end{array}$ & $\begin{array}{c}\text { Spacer Compressive } \\
\text { Yield Strength }\end{array}$ \\
\hline $\begin{array}{c}\text { Baseline } \\
\text { Temperature }\end{array}$ & $740 \#$ & $600-800 \mathrm{~F}$ & $-72,000 \mathrm{PSI}$ & $-116,000 \mathrm{PSI}$ & $\begin{array}{c}75,000 \text { PSI Min } \\
128,000 \text { PSI Typ }\end{array}$ & $\begin{array}{c}101,000 \mathrm{KSI} \text { Min } \\
150,000 \text { PSI Typ }\end{array}$ \\
\hline $\begin{array}{c}\text { Baseline +600 F } \\
\text { Temperature }\end{array}$ & $1,029 \mathrm{\#}$ & $1200-1400 \mathrm{~F}$ & $-100,000 \mathrm{PSI}$ & $-161,240 \mathrm{PSI}$ & $\begin{array}{c}85,000 \text { PSI Min } \\
138,000 \text { PSI Typ }\end{array}$ & $\begin{array}{c}112,000 \text { PSI Min } \\
162,000 \text { PSI Typ }\end{array}$ \\
\hline
\end{tabular}

Figure 6

\section{1st TURBINE BLADE ATTACHMENT}

\section{Slip Along Contact Surfaces}

-Positive Slip - Blade moves radially outward relative to disk

- Centrifugal pull of blade stretches lugs and compresses attachment ring

- Radial growth of disk opens slot width

- Negative Slip - Blade moves radially inward relative to disk

-Attachment rings hotter than live disk

\section{$\mathbf{R}$}
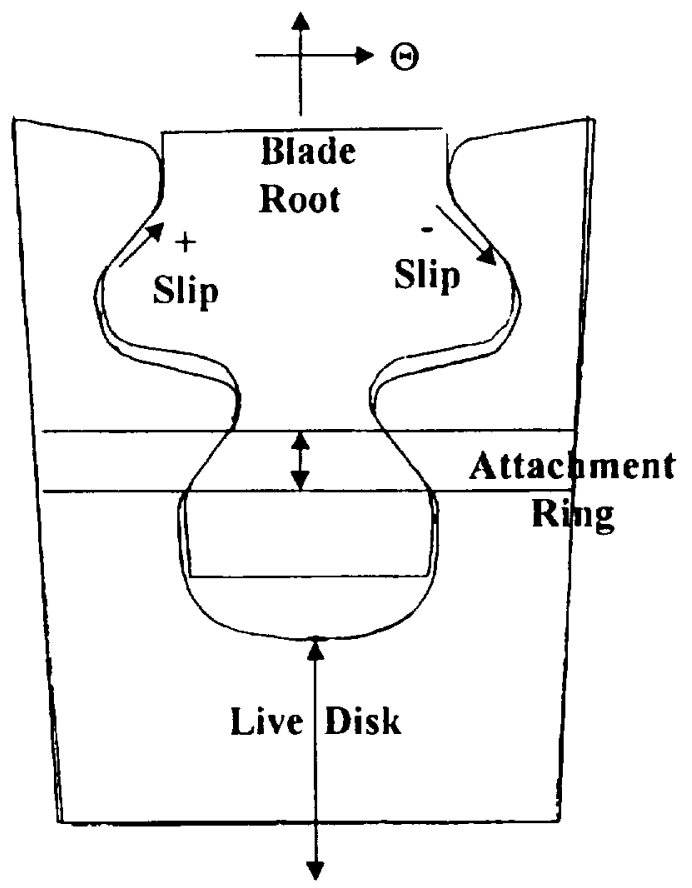

Figure 7 
1st TURBINE BLADE ATTACHMENT

Slip Along O.D. Contact Surface / No Friction

109 S/L Mission

2D Symmetrical Model

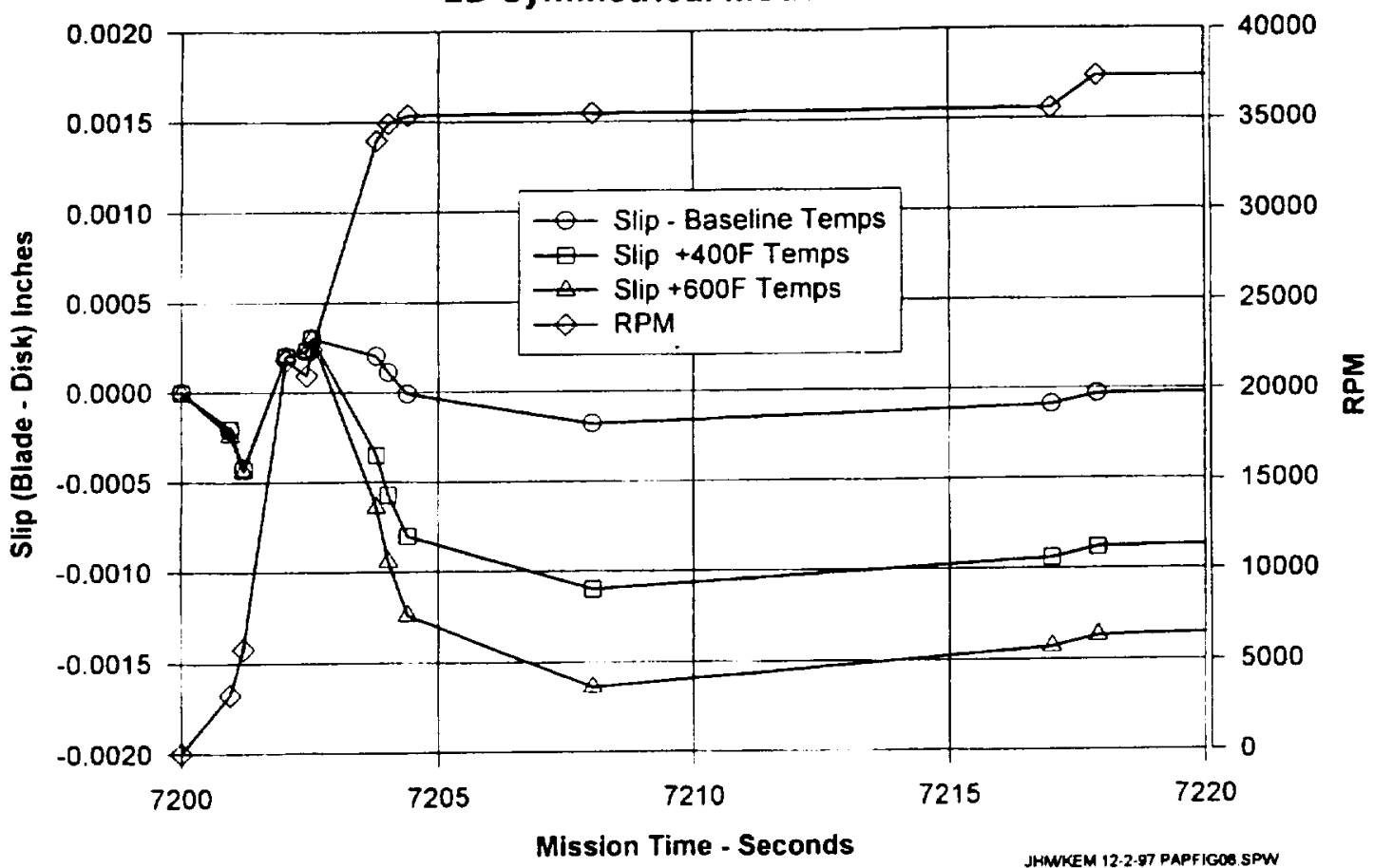

Figure 8

\section{1st TURBINE BLADE ATTACHMENT Contact Surface Loading With Friction}

-Summation of radial component of friction and normal force loads $=$ Blade Pull

- Blade slides outward

- $F f$ is inward

-Fn decreases

- Blade slides inward

-Ff is outward

-Fn increases

$$
\sum(\operatorname{Fr}(R)+F n(R))=\text { Pull }
$$

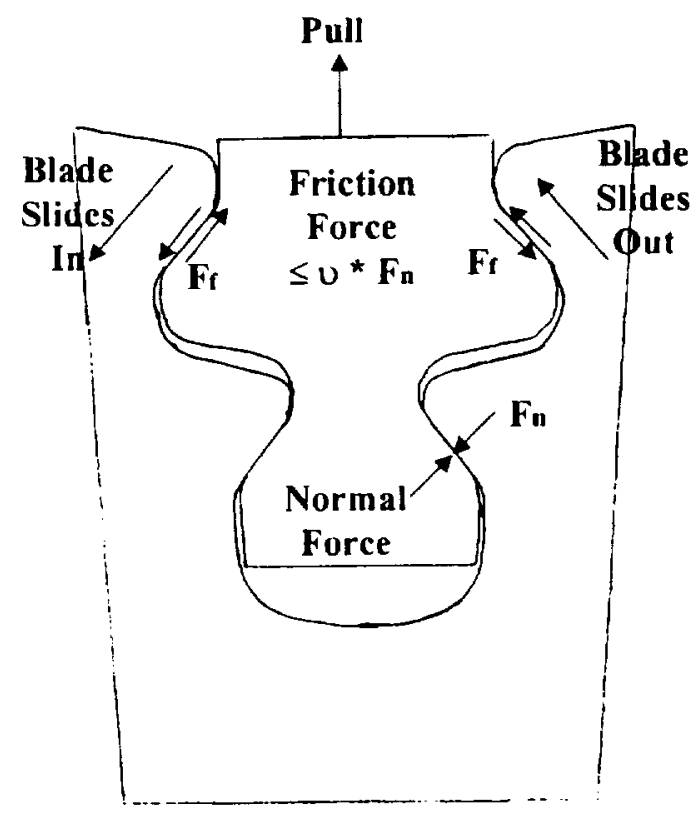

Figure 9

JIIM 11-18-98 PAPFIG9.PPT 
1st TURBINE BLADE ATTACHMENT

Slip Along O.D. Contact Surface

109 S/L Mission

2D Symmetrical Model

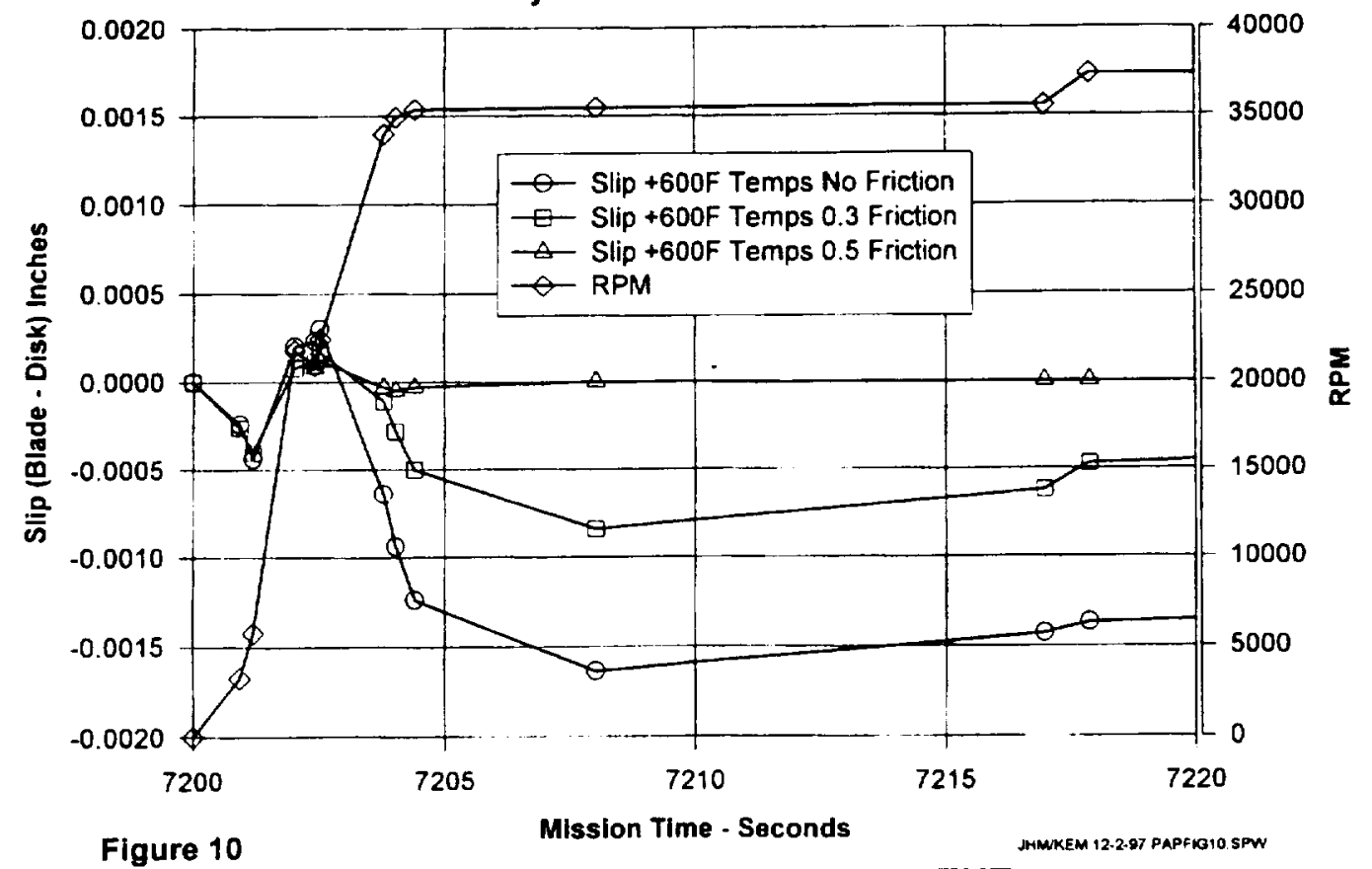

Figure 10

1st TURBINE BLADE ATTACHMENT

Contact Loading @ O.D. Tooth

B/L Temps 850F Max@ @/S

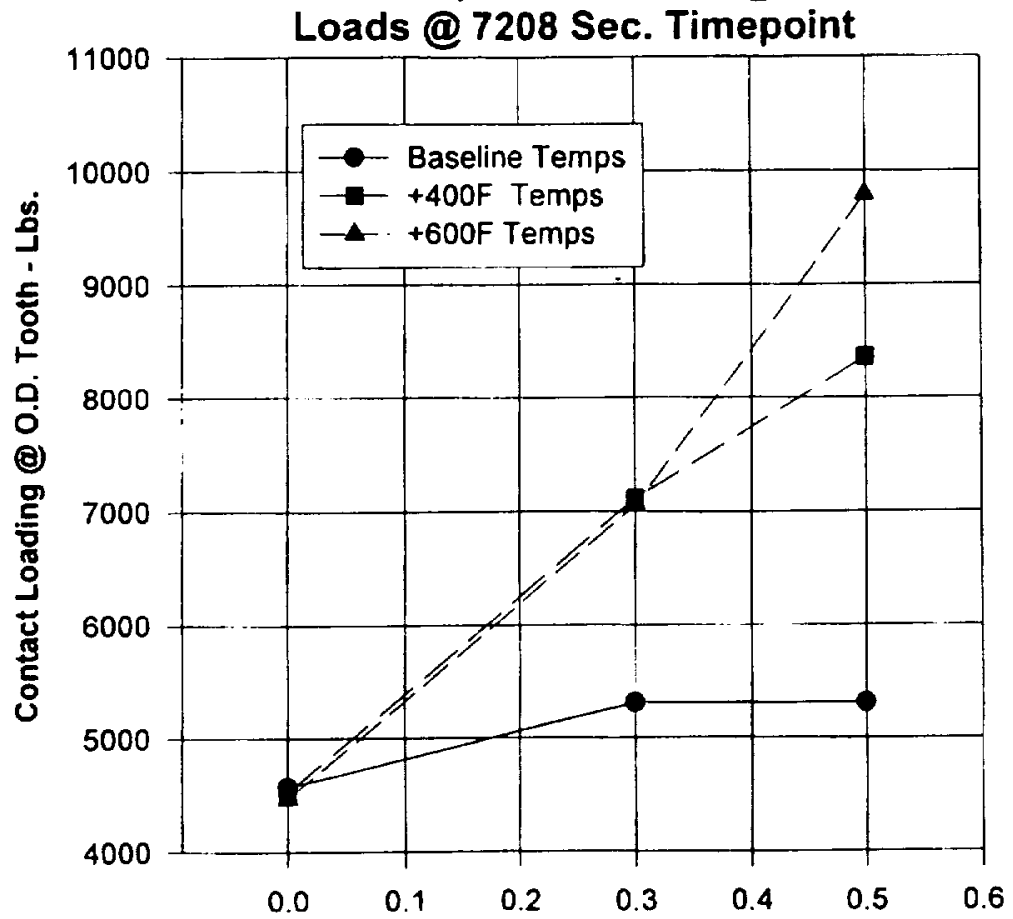

Friction Factor

JMWKEM 12-2-97 PAPFIG11 SPW

Figure 11 